flèches barbelées, ni des flèches empoisonnées, ni des traits enflammés. - Qu'étant sur un char, il ne frappe pas l'ennemi qui est à pied, ni le lâche, ni celui qui joint les mains, ni celui dont les cheveux sont déliés, ni celui qui est assis, ni celui qui s'écrie: «Je t'appartiens! ", - ni celui qui est endormi, ni celui qui n'a pas de cuirasse, qui est nu, qui est désarmé, ni celui qui regarde la bataille sans combattre lui-même, ni celui qui est aux prises avec un autre; - ni celui dont l'arme est brisée, ni celui qui est abattu, ni celui qui est grièvement blessé, - ni celui qui est épouvanté, ni celui qui a pris la fuite. C'est la loi deś guerriers vertueux.

Bhâgavata Purâna I. 7, 36 (trad. Burnouf) -- : Celui qui connaît la loi ne tue pas un homme ivre, un fou, un insensé, un idiot, un homme endormi, un enfant, une femme, ni un ennemi qui implore merci, ni celui dont le char est brisé, ni celui qui est glacé de frayeur.

\title{
Paul Oltramare.
}

\section{ITALIE}

LA CROIX ROUGE ITALIENNE

AUX GRANDES MANGUVRES DE L'ARMÉE ROYALE

dans le Haut-Novarais, du 28 août au 11 septembre 1880.

\section{Rapport général au Comite central.}

\section{Messievin,}

J'ai l'honneur de présenter au Comité central un rapport sur la participation de la Croix rouge italienne aux grandes manœuvres de 1880 , auprès du premier corps de l'armée, dans le Haut-Novarais.

Il appartient au commandant et au directeur sanitaire de la baraque-hopital des Chevaliers italiens de l'ordre de St-Jean de 
Jérusalem, de rédiger et de présenter des rapports détaillés sur le service sanitaire fait à Gattinara. Mais c'est à moi qu'incombe l'obligation de vous exposer, pour qu'ils soient connus aussi de toute l'Association, les résultats de cette première expérience partielle de la Croix rouge sur le champ des grandes manœurres, à un point de vue plus général et synthétique.

Comme représentant du Comité central, chargé spécialement par le Conseil de recueillir des observations exactes sur le mode de fonctionnement de la Croix rouge en campagne, je me rendis, le 27 aoùt à Gattinara, où, par ordre de S. E. le ministre de la guerre, la baraque-hôpital avait été placée. Plus tard, après m'être acquitté du devoir de me présenter au quartier-général pour faire acte de présence, témoignant par là de mon intention (comme notre représentant l'aurait fait en temps de guerre) de me mettre à la disposition du lieutenant-général commandant les grandes manœuvres, je suivis les sections du service de santé militaire pendant les mouvements de l'armée.

La présentation de la Croix rouge à l'armée et l'expérience de la baraque-hópital n'auraient pu se faire sous de meilleurs auspices. Un courant de sympathie et de bienveillance, parti du Ministre de la guerre, nous avait précédés et avait disposé les esprits en notre faveur.

Et ici je me hâte de dire que la conduite du commandant de la baraque et de tout le personvel fut admirable, et propre non-seulement à produire une bonne impression sur l'armée et sur la population, mais encore à concilier à l'institution de la Croix rouge la faveur et la bienveillance de tout le monde.

Le lieutenant-général Emilio Ferrero, commandant des grandes manœuvres, pénétré de l'importance morale qu'avait pour l'armée et pour le pays l'expérience qui se faisait sous ses ordres, facilita la tâche des Chevaliers qui, à ce mornent, représentaient la Croix rouge italienne, en les comblant de politesses et d'honneurs. Et toutes les troupes, depuis l'état-major général jusqu'au soldat, obéirent moins à un ordre qu'à un sentiment de bienveillance et en quelque sorte de protection collective, envers ce nouvel élément qui, soumis à une discipline et organisé presque militairement, suivait l'armée avec la mission de préparer dans son sein des secours civils, secours auxquels il parait que le soldat dédaigne de penser, mais qu'au- 
jourd'hui tout peuple civilisé est tenu de porter sur le champ de bataille.

Que la Croix ronge représente les droits de la civilisation comme correctif à ceux de la guerre, c'est ce qui apparaît clairement en campagne, alors que, mise sur le même pied que l'armée disposée en ordre de bataille, et tandis que le soldat obéit aux exigences impérieuses de la plus dure des lois sociales, on la voit conserver le caractère que lui a imposé sa haute mission et rester imperturbablement à son poste, pour servir uniquement les intérêts généraux de la civilisation, en même temps que l'intérêt particulier des citoyens qui combattent sous les drapeaux.

La position de la Croix rouge ainsi élablie sur un pied d'égalité avec celle de l'armée dans une bataille simulée, ne peut que s'accenluer plus vivement, dans le mème sens, lors d'une guerre véritable. Et, puisque j'ai à rendre compte des observations faites sur l'atlitude qu'ont prise sur le même terıain les deux éléments militaire et bourgeois, et sur les rapports qui ont existé entre eux, je dirai comment j'ai dù me convaincre de la nécessité absolue, pour nous, d'organiser et de règler les services de la Croix rouge en temps de guerre, avec des ordonnances el des formes qui se rapprochent le plus possible de l'organisation et de la discipline militaires.

La même impression favorable que fit sur l'armée la baraquehôpital, fut produite sans doute par son bon matériel et par la manière dont elle avait été disposẻe; mais tout cela aurait été à peine remarqué, ou du moins peu apprécié, si la direction n'avait pas été soumise à des ordonnances et à des règles strictement militaires.

L'autonomie militaire, cela est bien connu, ne supporte pas l'intrusion d'éléments hétérogènes, et la Croix rouge rencontrerait des difficultés insurmontables, ou du moins capables de compromeltre l'efficacité de son cuvre, si elle n'était pas subordonnée quasi-militairement et étroitement rattachée au commandement supérieur de l'armée en campagne.

Tout cela prouve toujours mieux que le Comité cental a agi sagement, en introduisant dans le statut fondamental de l'Association des dispositions propres à garantir, en temps de guerre, l'unité des forces de la Croix rouge, à l'instar de l'unité de l'armée, puis à la placer dans la dépendance du commandant en chef; cela prouve 
aussi que nous devons donner au personnel sanitaire et administratif des directions et une impulsion semblables à celles de l'élément militaire, auquel il doit s'associer en temps de guerre.

Passant des impressions générales aux observations sur l'activité pratique de la Croix rouge, dont le Comité central se préoccupe principalement, j'ai pu me persuader de l'évidente nécessité d'étudier un mode d'organisation de nos services, qui permette de diviser les forces saṇitaires de la Croix rouge en deux grandes catégories. L'une formerait notre première ligne, aurait un régime presque militaire, pourrait se mobiliser facilement et serait destinée à prendre la position qui nous serait prescrite sur les derrières de l'armée. L'autre, de seconde ligne, pourrait avoir des formes et un caractère plus bourgeois, serait échelonnée dans les villes et les bourgades situées sur le théâtre de la guerre, où elle organiserait des hòpitaux provisoires mais stables, pour soigner et surtout pour trier les malades et les blessés.

En troisième ligne nous trouverions le pays tout entier préparé, où nos comités sectionnaires seraient prompts à recueillir les ressources de la charité privée, et où un vaste réseau de sous-comités communaux est en train de se former, par les soins des syndics, pour organiser des secours dans toute l'étendue du royaume.

Nous devrions penser à organiser la continuité du service sanitaire au moyen de ce réseau, spécialement le long des chemins de fer, afin d'obtenir la dispersion la plus complète et la plus prompte des malades et des blessés, en les cantonnant dans les communes et dans les grands hôpitaux, laissant absolument le service des transports à nos comités et sous-comités communaux.

Il me semble que, sur ces bases, nous devrions entreprendre de nouvelles études, aux grandes manœuvres de l'année prochaine et des suivantes, puisque ce n'est que lá où des troupes se meuvent en granđes masses, là où elles prennent des allures guerrières, là où elles parcourent de grands espaces en déplaçant leur champ d'action, que l'on peut faire des études et résoudre les problèmes qui concernent la pratique de notre œuvre. Il faut bien se persuader que, néanmoins, la Croix rouge éprouvera toujours une grande difficulté à déterminer avec quelque précision son point de départ et le moment où elle devra commencer à agir. 
Il est vrai pourtant que la Croix rouge, ayant un rôle subordonné à celui du service de santé militaire, recevra du chef de l'armée des ordres précis sur le mode de distribution de ses forces; mais il n'est pas moins vrai que, devant être prête à exécuter ces ordres, elle doit connaître tout le développement qu'elle peut donner à sa propre mancuvre, en prévoir toutes les circonstances, être prête pour toutes les éventualités; par conséquent les commandants des forces de la Croix rouge doivent être en état de bien comprendre leur position en campagne, quelle que puisse être la distance du champ de bataille, afin d'exéculer les ordres de manière à ne jamais créer des embarras sur les derrières de l'armée des combattants, et d'accomplir en même temps avec régularité et efficacité sa propre mission.

Je dirai aussi que, quoiqu'il soit établi en principe que la Croix rouge doit se tenir à une très-grande distance du théâtre de l'action, nous devons cependant être prêts à savoir manœuvrer dans son voisinage, puisque l'imprévu de la guerre peut, dans certaines circonstances, conduire le commandant de l'armée à réclamer les services de notre première ligne, sur telle partie du champ des hostilités où les ambulances militaires, pour quelque raison de guerre, seraient empêchées d'accourir promptement.

Ces idées se sont présentées à mon esprit en parcourant le champ des manouvres, tantôt en suivant les détachements sanitaires et le travail du transport des blessés sinulés, tantôt en étudiant le terrain envahi ou abandonné alternativement par les corps d'armée, et plus fréquemment en observant ce qui se passail sur le terrain, sur les derrières des troupes en mouvement.

De nouvclles études pourront confirmer ou infirmer le résultat de mes observations, mais je me flatte que de toute manière elles pourront être utiles comme point de départ, lorsque nous discuterons notre règlement pour le temps de guerre.

Enfin, comme cela était bien naturel, j'ai porté plus particulièrement mon attention sur l'essai qui se faisait de la baraque-hôpital, pour voir quels résultats pratiques pouvait donner cette sorte de matériel nobile.

Je n'hésite pas à dire que l'expérience a répondu parfaitement aux prévisions, quant au matériel de la baraque et au service 
sanitaire, après que les difficultés du transport et de l'installation eurent été vaincues. Je crois que ce succès est dù en grande partie à une direction intelligente, à une discipline parfaite, et $\grave{a}$ l'abnégation des Chevaliers ainsi que de tout le personnel préposé au service hospitalier.

Mais j'ai été conduit à me former une opinion bien différente sur l'utilité des baraques-hòpitaux en tant que matériel mobile de campagne. Sous ce rapport, mon préavis est défavorable.

Cette espèce de matériel, quoique étant en fait transportable d'un lieu à un autre avec une mancuvre relativement facile, ne réalise pourtant en aucune manière la vraie mobilité, telle que la requièrent les exigences de la guerre.

Une baraque-hòpital, comme celle construite avec tant de perfection et de luxe d'aménagement par les Chevaliers italiens, capable de contenir de 60 à 100 malades, pourvue de tentes supplémentaires, de voitures de service, de provisions de bouche, de médicaments, d'équipements pour le personnel, ne pèserait pas moins de 30,000 kilogrammes, aurait besoin de huit wagons pour son déplacement, de 60 voitures pour être transportée du chemin de fer à la place où elle devrait être dressée, et de plusieurs journées de travail avant qu'on pût la dire en parfail état de service.

Tout cela veut dire qu'on peut la mettre ou l'on veut, qu'elle est entièrement autonome, réunissant ou portant avec elle tout ce qui est nécessajre à un hôpital en activité, qu'elle est susceptible de notables et faciles agrandissements sur place, mais que, du moment qu'elle fonctionne, elle n'a plus le mérite de la mobilité, et que personne ne songerait, vu la brièveté des guerres modernes, à la faire changer de position.

Ce caractère de stabilité joint aux considérations économiques, qui ne sont pas en rapport avec le nombre restreint de malades et surtout de blessés qu'elle peut recevoir, conduisent à un jugement négatif comme hôpital mobile de campagne, bien qu'en elle-même la baraque soit un modèle, non économique à la vérité, d'hôpilal provisoire.

Je ne saurais cependant, malgré le jugement que je viens de porter sur son compte, en contester l'utilité d'une manière absolue, vis-à-vis des services que la Croix rouge est appelée à rendre et du mode de notre organisation actuelle. 
Les baraques-hòpitaux, comme celle que nous avons expérimentẻe, ont et peuvent conserver une sorte d'autonomie, qui convient parfaitement au caractère et au tempérament des corps moraux que nous appelons à prêter leur concours éminemment bienfaisant dans le sein même de notre grande association. Si d'autres corps moraux, à l'imitation des Chevaliers italiens, ou si les Chevaliers eux-mêmes construisaient d'autres baraques-hôpitaux, en s'engageant à les employer pour le service en temps de guerre, elles pourraient être très-utilement dressées comme hôpitaux de stations, spécialement là où on en improvise sur les chemins de fer, au croisement des grandes lignes, où manqueraient les moyens d'abriter les blessés qui attendraient le moment de l'évacuation, el de soigner ceux dont l'état se serait aggravé pendant le trajet.

Parmi les arguments qui plaident contre l'exclusion absolue des baraques-hòpitaux pour le service sanitaire de la Croix rouge sur le théâtre de la guerre, on peut invoquer la possibilité d'interpréter en leur faveur l'article 4 de la Convention de Genève, en vertu duquel les ambulances ne sont pas soumises aux lois de la guerre. Mais pour que les baraques-hòpitaux puissent être considérées comme de véritables ambulances, peut-être devraient-elles être accompagnées des voitures nécessaires à leur transport complet, ce qui leur conserverait un caractère de mobilité; elles devraient être véritablement transportables dans le cas visé par l'article 4 de la Convention, et figurer comme matériel d'ambulance déchargé pour fonctionner sur le terrain.

Nous ne pouvons pourtant pas dissimuler que la possession de cette sorte de matériel transportable nous obligerait à des prévisions difficiles à faire, puisque nous devrions, au premier symptôme d'hostilités, le concentrer sur les lignes ferrées du théâtre éventuel de la guerre, car il n'est pas vraisemblable que l'on aurait la possibilité de le transporter une fois le mouvement des troupes commencé.

Enfiu, sur ce genre de matériel de secours, je crois pouvoir conclure, en me foudant sur les données de l'expérience recueillies avec le plus grand soin :

Que les baraques-hôpitaux ne peuvent être utilement mises en œuvre que comme hôpitaux provisoires de stations.

Qu'on devrait les exclure du matériel préparé directement par le Comilé central et par les Comités sectionnaires. 
Que pour en obtenir une aide efficace, non-seulement leur construction et leur entretien, mais tout leur service intérieur devrait en tous cas être confié aux corps moraux, agrégés à la Croix rouge et possédant un personnel particulier, capable et discipliné.

D'autres études de détail et d'autres observations, qui ne peuvent trouver place dans un rapport général, seront exposées par moi à la Commission technique, quand elle sera appelée à discuter sur le matériel mobile et particulièrement sur le projet de tentes, applicables aux voitures, que j'ai étudiées à Gattinara et que je présenterai au Conseil.

Je voudrais pouvoir décrire maintenant les sections? du service de santé militaire, et surtout parler de la nouvelle voiture pour les hommes atteints de blessures ou de maladies graves, imaginée par l'éminent capitaine médical Salvatore Guida, représentant du Ministère de la guerre dans le Comité central, et construite sous sa direction. J'ai trouvé cette voiture très-pratique et la mieux réussie de toutes celles que j'ai eu jusqu'à présent l'occasion d'examiner, en sorte que nous pourrions la prendre pour modèle et. l'adapter à nos besoins; mais je m'abstiens d'entrer dans les détails de ce qui regarde le service de santé militaire, afin de ne pas outrepasser les limites de ma compétence et celles de ma mission.

Avant de terminer mon rapport, je dois encore rendre compte d'un grand résultat, d'ordre moral, qui me semble avoir été obtenu expérimentalement et que vous apprendrez avec plaisir.

Notre principal objectif à Gattinara était certainement de faire connaître à notre valeureuse et chère armée l'institution de la Croix rouge. Eh bien ! le résultat a été tel que vous pouviez le désirer.

La présence de la Croix rouge a produit sur le camp une excellente impression, qui démontre toujours plus la bienfaisante action que la Croix rouge exerce sur les troupes, et son incontestable influence sur le moral des armées.

Sous l'emblème de la Croix rouge, les soldats voient le cœur de la nation, qui palpite pour eux et qui, au moment du danger, détache d'elle-même une autre armée, celle des soldats de la charité, prompte à secourir ceux qui tombent pour la défense du pays.

Telles sont, Messieurs, les impressions que j'ai reçues et les observations 'que j'ai recueillies en suivant les grandes manœuvres, 
et en étudiant attentivement ce qui se rapporte à l'œuvre de la Croix rouge.

D'aulres, plus compétents que moi, y ajouteront de nouvelles études et de nonvelles observations, s'il nous est permis de renouveler ces expériences sur le champ de grandes manœuvres. J'ai fait ce qu'il était en mon pouvoir de faire, en m'acquittant avec beaueoup d'intérêt et avec zèle de l'honorable mandat que vous m'aviez confié.

Le Comité central reconnaîtra combien il lui a été utile d'entrer dans une voie pratique, combien le Comité des Chevaliers y a brillamment contribué, quelle reconnaissance l'Association doit au gouvernement du roi, qui en admettant la Croix rouge italienne à participer aux grandes mancuvres, nous a déjà jugés dignes de prendre place à còté de notre glorieuse armée.

Le Secrélaire-génèral,

Vincenzo Maggiorani.

\section{NORWEGE}

PRÉSIDENCE DU COMITÉ GENTRAL

Au Comité international de la Croix rouge.

Christiania, le 18 février 1881.

Monsieur le Président,

C'est avec grand regret que nous avous l'honneur de vous communiquer que le ministre d'Etat Stang, président de notre direction depuis 1866 , alors qu'il invita ses compatriotes à s'associer au Comité norwégien de secours aux militaires blessés, a été obligé de renoncer à ses fonctions à cause de sa santé affaiblie.

Nous ajouterons que le Comité a été assez heureux pour décider 